\title{
Hand Foot and Mouth Disease (HFMD) in a 3 Years Old Boy in Bangladesh: A Case Report
}

\author{
Arifa Akram ${ }^{1}$, Lubana Akram²
}

\begin{abstract}
${ }^{1}$ Medical Officer, Department of Virology, Institute of Epidemiology, Disease Control and Research, Dhaka, Bangladesh; ${ }^{2}$ Medical
\end{abstract} Officer, Adhunik Sadar Hospital, Natore, Bangladesh

[Received: 7 March 2019; Accepted: 20 May 2019; Published: 1 December 2019]

\section{Abstract}

Hand, foot \& mouth disease (HFMD) is a contagious and emerging infectious disease caused by picorna viridae family. It was first reported in New Zealand in 1957 is caused by Coxsackie virus A16 (CVA16), human enterovirus 71 (HEV71) and occasionally by Coxsackie virus A6 and Coxsackie virus A10, are also associated with HFMD and herpangina. Though only small scale outbreaks have been reported from United States, Europe, Australia Japan and Brazil for the first few decades, since 1997 the disease has noticeably changed its character as noted in different Southeast Asian countries. In recent years Bangladesh also faces some cases of HFMD. Though HFMD is a mild disease but in rare cases may develop neurological complications. Early detection and good clinical assessment can prevent the fatal progression and also can reduce morbidity and mortality regarding HFMD. [Bangladesh Journal of Infectious Diseases, December 2019;6(2):53-55]

Keywords: Hand-foot-mouth disease; Coxsackie virus; Entero virus; Children

Correspondence: Dr. Arifa Akram, Medical Officer, Institute of Epidemiology, Disease Control and Research, Mohakhali,

Dhaka, Bangladesh; Cell no.: +8801816296249; Email: drbarna43@gmail.com

Conflict of interest: There is no conflict of interest to any of the authors of this article.

Funding agency: The study was not funded by any authority.

Contribution to authors: Akram A, Akram Lcontributed in disease detection. Akram A had prepared and revised the paper.

How to cite this article: Akram A, Akram L. Hand Foot and Mouth Disease (HFMD) in a 3 Years Old Boy in Bangladesh: A Case Report. Bangladesh J Infect Dis 2019;6(2):53-55

Copyright: (02019. Akram and Akram. Published by Bangladesh Journal of Infectious Diseases. This article is published under the Creative Commons CC BY-NC License (https://creativecommons.org/licenses/by-nc/4.0/). This license permits use, distribution and reproduction in any medium, provided the original work is properly cited, and is not used for commercial purposes.

\section{Introduction}

Hand-foot-mouth disease (HFMD) is a common viral illness that commonly affects children younger than 5 years ${ }^{1}$.It is highly contagious and the clinical manifestations include mild fever with a characteristic vesicular eruption on the hands, feet, buttock and oral cavity ${ }^{2}$. HFMD occurs mainly by serotype of entero virus most frequently Coxsackie virus A16 (CAV16) and human entero virus 71 (HEV71). Other serotypes causing outbreaks of
HFMD are coxsackie A viruses 5, 6, 7, 9, and 10; coxsackie B viruses 2 and $5^{3}$. This disease was first reported in New Zealand in 1957 and then emerged in the Asia-Pacific region in mid-1990s ${ }^{4}$.Outbreaks of HFMD in Malaysia occur periodically. In other Asian countries such as China, Taiwan, Singapore and Vietnam, outbreaks of this disease also occur, leading to a number of fatalities ${ }^{5}$.

During summer and early autumn most of the cases occur. Commonly, this is a mild self-limiting 
illness. Without scarring the skin lesions heal spontaneously ${ }^{6}$. In rare cases, patients may also develop neurological complications, such as encephalomyelitis, aseptic meningitis, and acute flaccid paralysis ${ }^{7}$.

\section{Case Presentation}

A three years old boy reported to the Natore district hospital in the month of October, 2017 with the complaint of difficulty in swallowing since a day. His mother gave history of fever since 2 days. The patient had developed fever all of a sudden with no other symptoms. After 1 day his mother had noticed two papules; one in the leg and one in the buttock and difficulty in swallowing. The patient experienced a itching over the rashes. Informed consent was obtained from the parent as a part of the routine protocol before the clinical examination. On examination, the patient was feverish and temperature was of $102^{\circ} \mathrm{F}$. Many papules were noted on the right leg over knee joint and buttock (Fig. 1 and 2). Buccal cavity revealed a vesicular lesion about $2 \mathrm{~mm}$ on left side. Based on the above clinical features, the case was diagnosed as hand, foot and mouth disease.

The mother of the baby was advised to take plenty of liquids. To control fever and reduce itching, he was prescribed to take paracetamol and antihistamine syrup. After three days, most of the papules had turned into fluid filled blisters. However, patient's condition was improved after improvement of palatal lesions and blisters. The patient was followed up for 6 months and no recurrence was noted. Other diagnoses to be considered were herpangina, aphthous ulcers, varicella, gingivostomatitis, erythema multiforme, and drug eruption ${ }^{2}$.

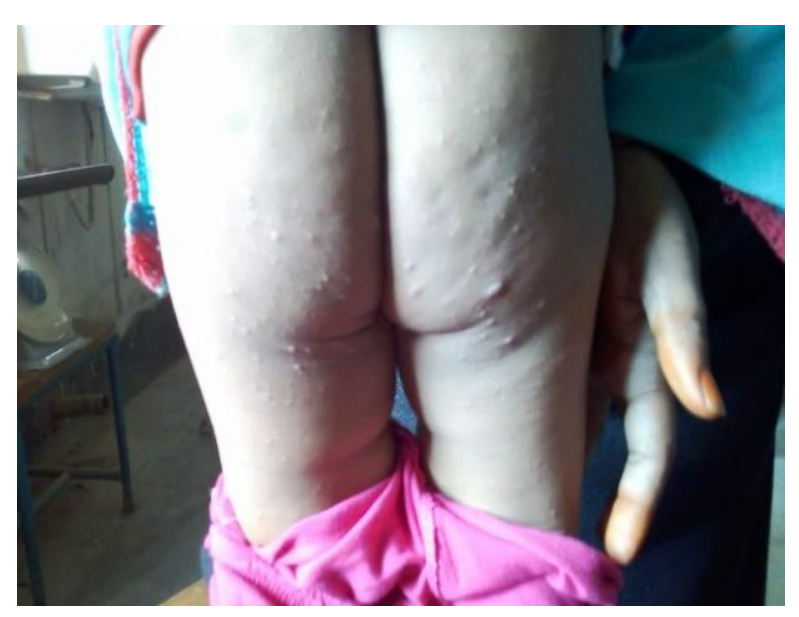

Figure 1: Papules seen in buttock

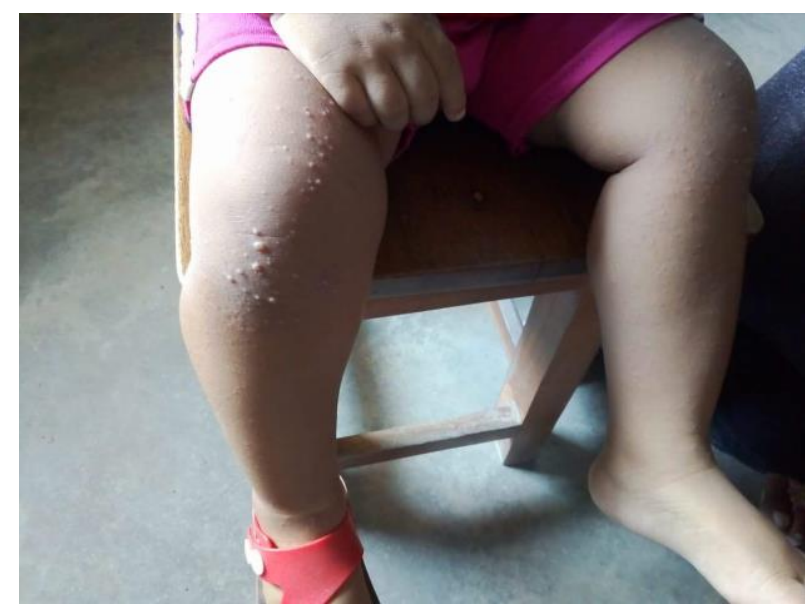

Figure 2: Papules seen over knee joint

\section{Discussion}

Hand, foot, and mouth disease (HFMD) belongs to the genus Entero virus in the Picorna viridae family, most commonly coxsackie virus A16 and entero virus $71^{2}$.Enteroviruses are positive sense single stranded RNA viruses. HFMD has also been reported to be caused by coxsackie virus A5, A6, A7, A9, A10, B2 and B5 strains ${ }^{1}$.

The disease is manifested by the sudden appearance of erythematous papulo vesicular eruptions, fever and malaise after an incubation period of 3 to 7 days which is highly contagious. Vesicles are round or oval which appear in crops and persist in groups over hand, feet, perioral area, knees, buttocks and also oral region generally. In the palms and soles where skin is thick may not develop classical vesicle; they may instead persist as erythematous papules. Without any complication the disease usually improves spontaneously after 7-10 days. In severe disease, cardio respiratory and neurological involvement may develop ${ }^{7}$.

The virus spreads to other people through saliva, feces, vesicular fluid, respiratory secretions, and respiratory droplets. Close contact with HFMD patients and poor personal hygiene are risk factors for HFMD. HFMD occurs during the summer in temperate regions but at any time in tropical countries. Interestingly, our patient presented in September and October. Our patient presented with severe pain over the hand and buttock and also difficulty in swallowing. Usually symptoms resolve in 7-10 days. However, it can present with atypical features such as vesicles or papules on the face or bullae on the trunk, and resemble eczema herpeticum or chickenpox. Onychomades is can occur 1-2 months following infection. Oral lesions 
usually appear simultaneously with or precede cutaneous lesions, but the simultaneous occurrence of lesions on the hands, feet and in the oral cavity in adults is very infrequent ${ }^{1}$.

The diagnosis of HFMD is typically based on clinical grounds and laboratory studies are usually unnecessary ${ }^{7}$. Although, this shares some clinical resemblance with other diseases like varicella zoster, papular urticaria, impetigo, drug eruptions, vasculitis, dermatitis and pompholyx, the constellation of features are unique enough to aid instant clinical diagnosis with certainty in almost all cases ${ }^{6}$.If diagnosis is unclear, then a multiplex reverse transcriptase-polymerase chain reaction (RT-PCR) assay can be used as a rapid and costeffective diagnostic tool to detect the presence of HFMD $^{1}$.

There is neither an effective antiviral therapy, nor an effective vaccine available against the disease. This is a contagious disease and has the potentiality to spread very fast over a large population in the community. Treatment for HFMD is mainly symptomatic. To control fever, antipyretics are given and to reduce itching antihistamines are given. Prevention of further spread of the disease is the only way to control a disease from becoming a large outbreak. While the organisms are enterovirus, they spread through oro-faecal route. Restriction of the affected children from attending school or other outdoor activities is a very simple but effective strategy ${ }^{7}$.

The Centers for Disease Control and Prevention recommend all affected patients and their close contacts to practice the following measures: hand washing after changing diapers and using toilets; frequent disinfection of commonly used surfaces and objects; avoiding close contact with infected individuals. These recommendations are simple tasks that play an important role in preventing further spread of infection ${ }^{1}$.

\section{Conclusions}

Hand, foot, and mouth disease (HFMD) is commonly seen in children and immune compromised adults. HFMD has been emerging as a common benign human childhood disease of selflimiting nature in the last few years, but previously considered a disease of cattle. The incidence of this disease increases every year. Although in most of the cases, it is nonfatal, there are some reported cases of complications seen in HFMD patients. For this reason this has got less consideration from the medical group, researchers, public health department and policy makers. All physicians should be aware of the symptoms of this disease and possible complications.

\section{References}

1. Centers for Disease Control and Prevention (CDC). Hand, foot and mouth disease. Available on https://www.cdc.gov/hand-foot-mouth/index.html. Accessed on July 17, 2018.

1. Andreoni AR, Colton AS. Coxsackievirus B5 associated with hand-foot-mouth disease in a healthy adult. JAAD case reports. 2017 Mar 1;3(2):165-8.

2. Sah VK. Hand, Foot and Mouth Disease: A case report. Janaki Medical College Journal of Medical Science.;4(1):59-64 3. Sarkar PK, Sarker NK, Tayab MA. Hand, Foot and Mouth Disease (HFMD): An Update. Bangladesh Journal of Child Health. 2017 Feb 13;40(2):115-9

4. Koh WM, Badaruddin H, La H, Mark I, Chen C, Cook AR. Severity and burden of hand, foot and mouth disease in Asia: a modelling study. BMJ global health. 2018 Jan 1;3(1):e000442 5. Kumar K B, Kiran A G, Kumar B U. Hand, foot and mouth disease in children: A clinico epidemiological study. Indian $\mathbf{J}$ Paediatr Dermatol 2016;17:7-12.

6. Kashyap RR, Kashyap RS. Hand, foot and mouth disease-a short case report. Journal of clinical and experimental dentistry. 2015 Apr;7(2):e336

7. Yacoub AT, Nanjappa S, Gajanan G, Bohra C, Greene JN. Hand, foot, and mouth disease: An emerging disease in immunocompetent adults. International Journal of Case Reports and Images (IJCRI). 2016 Oct 28;7(12):805-9 